\title{
THE LEVEL SETS OF THE MODULI OF FUNCTIONS OF BOUNDED CHARACTERISTIC
}

\author{
BY
}

ROBERT D. BERMAN

\begin{abstract}
For $f$ a nonconstant meromorphic function on $\Delta=\{|z|<1\}$ and $r \in$ (inf $|f|$, sup $|f|)$, let $\varrho(f, r)=\{z \in \Delta:|f(z)|=r\}$. In this paper, we study the components of $\Delta \backslash E(f, r)$ along with the level sets $E(f, r)$. Our results include the following: If $f$ is an outer function and $\Omega$ a component of $\Delta \backslash \mathscr{L}(f, r)$, then $\Omega$ is a simply-connected Jordan region for which (fr $\Omega) \cap\{|z|=1\}$ has positive measure. If $f$ and $g$ are inner functions with $\mathcal{E}(f, r)=\ell(g, s)$, then $g=\eta f^{\alpha}$, where $|\eta|=1$ and $\alpha>0$. When $g$ is an arbitrary meromorphic function, the equality of two pairs of level sets implies that $g=c f^{\alpha}$, where $c \neq 0$ and $\alpha \in(-\infty, \infty)$. In addition, an inner function can never share a level set of its modulus with an outer function. We also give examples to demonstrate the sharpness of the main results.
\end{abstract}

1. Introduction. Let $\Delta=\{|z|<1\}$ and $C=\{|z|=1\}$. For $f$ a nonconstant meromorphic function on $\Delta$ and $r \in(\inf |f|$, sup $|f|)$, let $\mathcal{L}(f, r)$ denote the level set $\{|f|=r\}$. In this paper we study $\mathscr{L}(f, r)$ along with the components of $\Delta \backslash \mathcal{L}(f, r)$ (i.e., the components of $\{|f|>r\}$ and $\{|f|<r\}$ ), when $f$ is in the class $\mathscr{N}$ of all nonconstant analytic functions of bounded Nevanlinna characteristic.

In $\S 2$, definitions and background facts are given. Two subclasses of $\mathscr{N}$ defined in this section are of particular interest, the Smirnov class $\mathfrak{T}^{+}$(cf. Definition 2.3), and the class of inner functions 9 (cf. Definition 2.4).

In $\S 3$, the components of $\{|f|>r\}$ are considered. The main result is Theorem 3.2. It asserts that if $f \in \mathcal{R}^{+}$and $\Omega$ is a component of $\{|f|>r\}$, then ( $\left.\operatorname{fr} \Omega\right) \cap C$ has positive measure. It is also shown that if $f \in \mathcal{G}$, then almost all radii ending in ( $\operatorname{fr} \Omega) \cap C$ are eventually contained in $\Omega$ (Theorem 3.3).

In $\S 4$, the components of $\{|f|<r\}$ are taken up. The starting point is a result of Lohwater [8, pp. 5-7]: If $f$ is a nonvanishing inner function, then a component $\Omega$ of $\{|f|<r\}$ has the properties (i) $\Omega$ is a simply-connected Jordan region, and (ii) ( $\mathrm{fr} \Omega) \cap C$ is of zero measure. Theorem 4.1, which is based on a result of MacLane [9, Theorem 1] and Theorem 3.2, asserts that property (i) holds for all functions in the class $\mathfrak{\pi}^{+}$. Concerning property (ii) we prove two results. Theorem 4.2 characterizes the class of functions in $\mathcal{X}$ for which (ii) holds as the class $\{c f: f \in \mathscr{G}, c \neq 0\}$. Theorem 4.3 shows that this property is sharp in the following sense. If $F$ is a nonempty closed set of measure 0 in $C$ and $r \in(0,1)$, then there exists a nonvanishing inner function $f$ along with a component $\Omega$ of $\{|f|<r\}$ such that $($ fr $\Omega) \cap C=F$.

Received by the editors November 19, 1982.

1980 Mathematics Subject Classification. Primary 30D50, 30D40.

Key words and phrases. Level sets, bounded characteristic, inner functions, Smirnov class.

(C) 1984 American Mathematical Society $0002-9947 / 84 \$ 1.00+\$ .25$ per page 
In $\S 5$, the compactness of $\mathcal{E}(f, r)$ and its components is studied. In Theorem 5.1 two classes of functions are determined, the class of nonconstant analytic functions $f$ for which every level set $\mathscr{L}(f, r)$ is compact, and the class of $f$ in $\mathscr{T}$ for which all of the components of $\mathcal{L}(f, r)$ are compact for every $r$. Each consists of the nonzero constant multiples of a class of Blaschke products.

The final section, $\S 6$, is devoted primarily to level set uniqueness theorems. Theorem 6.1 is the basis for all of them. It asserts that for $f$ and $g$ in 9 , we have $\mathcal{L}(f, r)=\mathcal{L}(g, s)$ if and only if $f$ has an analytic $\alpha$ th power $f^{\alpha}$ for $\alpha=(\log s) / \log r$, and $g=\eta f^{\alpha}$ for some $\eta \in C$. This leads to Theorem 6.3 which states that if $f \in q$ and $g$ is an arbitrary nonconstant meromorphic function on $\Delta$ satisfying $\mathcal{L}\left(f, r_{k}\right)=$ $\mathcal{L}\left(g, s_{k}\right), k=1,2\left(r_{1} \neq r_{2}\right)$, then $g=c f^{\alpha}$ where $\alpha \in(-\infty, \infty), f^{\alpha}$ is a meromorphic $\alpha$ th power of $f$, and $c$ is a nonzero complex constant. Corollary 6.3, a corollary to the proof of Theorem 6.3, is stated for nonconstant meromorphic functions $f$ and $g$, one of which, say $f$, has a zero. It states that if $\mathcal{L}\left(f, r_{n}\right)=\mathcal{L}\left(g, s_{n}\right)$ for a sequence $\left(r_{n}\right)_{1}^{\infty}$ converging to 0 , then $g=c f^{\alpha}$, where $\alpha \in(-\infty, \infty)$ and $c \neq 0$. Example 6.1 shows that this result is not valid when the assumption that $f$ has a zero is replaced by $\inf |f|=0$. Among other results in this section, we prove that a nonconstant inner function can never share a level set of its modulus with an outer function.

At this time, the author wishes to thank Professors Maurice Heins and Marlies Gerber for helpful discussions they had with him. In addition, we note that $\mathrm{K}$. Stephenson and C. Sundberg have independently proved a result closely related to Theorem 6.1.

2. Definitions and background results. We start by stating without proof a standard result concerning the local behavior of nonconstant meromorphic functions defined on a region in the complex plane $\mathbf{C}$.

PROPOSITION 2.1. Let $f$ be a nonconstant meromorphic function which is of multiplicity $n$ at $a \in \mathcal{L}(f, r)$. Let $l_{k}$ be the intersection of $\Delta$ with the line passing through the origin at an angle $(\pi k) / n$ with the real axis, for $k=0, \ldots, n-1$. Then there exists a univalent analytic map $\psi$ of $\Delta$ onto a neighborhood $N$ of $a$, such that $\psi(0)=a$, $N \cap \mathcal{L}(f, r)=\psi\left(\cup_{0}^{n-1} l_{k}\right)$, and the component open sectors of $\Delta \backslash \cup_{0}^{n-1} l_{k}$ are mapped by $\psi$ alternately into $\{|f|<r\}$ and $\{|f|>r\}$.

The following theorem is a consequence of the maximum principle, Proposition 2.1 , and the fact that the only 1 -manifolds (with a countable basis) are, up to homeomorphism, the reals $R$ and the unit circle $C$. In what follows $\bar{X}$, resp. $\operatorname{fr} X$, is used to denote the closure, resp. frontier, in the extended complex plane $\hat{\mathbf{C}}$, of $X \subseteq \hat{\mathbf{C}}$. For $X \subseteq Y \subseteq \hat{\mathbf{C}}$, the set $X$ is said to be a relatively compact subset of $Y$ if $\bar{X} \subseteq Y$.

THEOREM 2.1. Let $f$ be a nonconstant analytic function on $\Delta$ and $r \in \inf |f|$, $\sup |f|)$. Let $\Omega$ be a component of $\{|f|<r\}$. The following assertions are valid.

(i) $\Omega$ is simply-connected.

(ii) Except for the at-most countable set of points in (fr $\Omega) \cap \Delta$ where $f$ has multiplicity $>1$, we have $(\operatorname{fr} \Omega) \cap \Delta$ is locally a regular analytic Jordan arc which is 
contained in the frontier of exactly two components of $\Delta \backslash \mathcal{L}(f, r)$, one $\Omega$ and the other a component of $\{|f|>r\}$.

(iii) A component l of ( $\mathrm{fr} \Omega) \cap \Delta$ is homeomorphic to $R$ or $C$.

(iv) A component l of ( $\mathrm{fr} \Omega) \cap \Delta$ is homeomorphic to $C$ if and only if $\Omega$ is a relatively compact subset of $\Delta$. In this case, $\Omega$ is the bounded component of $\hat{\mathbf{C}} \backslash l$.

In connection with the proof of (iii), we make the following observations. It is necessary to show that $l$ is locally homeomorphic to $R$ at a point $a \in l$ where the multiplicity of $f$ is greater than 1. This is accomplished by applying Proposition 2.1 and showing that $l \cap N$ is, in fact, the image under $\psi$ of the radii bounding a component open sector of $\Delta \backslash \cup_{0}^{n-1} l_{k}$. If this were not the case, then using Proposition 2.1, we see that the component of $\{|f| \leqslant r\}$ containing $\Omega$ encircles a component of $\{|f|>r\}$ which is relatively compact in $\Delta$. This contradicts the maximum principle.

The following is a corollary of Theorem 2.1(iii) and the continuity of $f$.

COROllaRy 2.1. Let $l$ be a noncompact component of $(\mathrm{fr} \Omega) \cap \Delta$ and let $\gamma$ be a homeomorphism of $(-1,1)$ onto l. Then $\lim _{t \rightarrow \pm 1}|\gamma(t)|=1$.

We proceed now to define classes of functions on $\Delta$ and state some relevant facts about them.

DEFINITION 2.1. The MacLane class $\mathcal{L}$ is the class of nonconstant analytic functions $f$ on $\Delta$ such that for each $r \in(\inf |f|$, sup $|f|)$ we have $\lim _{t \rightarrow 1} S_{r}(t)=0$, where

$$
S_{r}(t)=\sup [\operatorname{diam} l: \text { l a component of }\{t<|z|<1\} \cap \mathcal{L}(f, r)], \quad t \in(0,1) .
$$

By definition, $\operatorname{diam} W=\sup \{|z-w|: z, w \in W\}$ for $W$ a subset of the complex plane C. The observations of MacLane (cf. [9, p. 9]) show the following.

Proposition 2.2. If $f \in \mathcal{L}$ and $\gamma$ is a continuous mapping of $[0,1)$ into some level set $\mathcal{L}(f, r)$ satisfying $\lim _{t \rightarrow 1}|\gamma(t)|=1$, then $\lim _{t \rightarrow 1} \gamma(t)$ exists.

The next proposition is a consequence of Proposition 2.1, which insures that the components of a level set $\mathcal{L}(f, r)$ cannot cluster at a point of $\Delta$, and Definition 2.1.

Proposition 2.3. If $f \in \mathcal{L}$ and $\left(l_{k}\right)_{1}^{\infty}$ is a sequence of components of $\mathcal{L}(f, r)$, then $\lim _{k \rightarrow \infty} \operatorname{diam} l_{k}=0$.

The following is part of [9, Theorem 1].

THEOREM 2.2 (MACLANE). The class 2 contains all nonconstant analytic functions on $\Delta$ having asymptotic values in a dense subset of $C$.

Recall that a function $f$ mapping into the extended plane $\hat{\mathbf{C}}$ is said to have an asymptotic value $w \in \hat{\mathbf{C}}$ at $\eta \in C$ if there exists a continuous map $\gamma$ of $[0,1)$ into $\Delta$ such that $\lim _{t \rightarrow 1} \gamma(t)=\eta$ and $\lim _{t \rightarrow 1} f \circ \gamma(t)=w$.

For the definitions in the remainder of this section along with the facts which are quoted concerning them, see Chapter 2 of P. Duren's book Theory of $H^{P}$ spaces [3]. 
For convenience, we shall only consider classes of nonconstant functions. The notation $\log ^{+}|z|$ is used for $\max \{\log |z|, 0\}, z \in \mathbf{C}$.

DEFINITION 2.2. The class $\mathfrak{X}$ of functions of bounded Nevanlinna characteristic is the class of all nonconstant analytic functions $f$ on $\Delta$ for which

$$
\int_{0}^{2 \pi} \log ^{+}\left|f\left(s e^{i \theta}\right)\right| d \theta
$$

is bounded for $0 \leqslant s<1$.

We state two theorems concerning this class.

TheOREM 2.3. For each function $f \in \mathcal{O}$, the radial limit $f^{*}(\eta)$ exists for almost all $\eta \in C$, and $\log \left|f^{*}\left(e^{i \theta}\right)\right|, \theta \in[0,2 \pi]$, is integrable.

Here and in the sequel, when $f$ is a real-valued or complex-valued function on $\Delta$, we denote the radial $\operatorname{limit}_{\lim _{t \rightarrow 1}} f(t \eta)$ by $f^{*}(\eta)$, for each $\eta$ in $C$ where the limit exists, finite or infinite. Note that Theorems 2.2 and 2.3 imply that $\mathfrak{R} \subset \mathcal{E}$.

The second result concerning $\mathfrak{N}$ is the canonical factorization theorem, Theorem 2.4 below. Before stating it, we recall some definitions that will be needed. A Blaschke product is a function of the form

$$
z^{m} \prod_{1}^{n}\left(\bar{a}_{k} /\left|a_{k}\right|\right) L_{a_{k}}(z), \quad z \in \Delta
$$

or

$$
z^{m} \prod_{1}^{\infty}\left(\bar{a}_{k} /\left|a_{k}\right|\right) L_{a_{k}}(z), \quad z \in \Delta,
$$

where $m, n$ are nonnegative integers, $\left(a_{k}\right)_{1}^{\infty}$ is a sequence taking values in $\Delta \backslash\{0\}$ and satisfying the Blaschke condition $\Sigma\left(1-\left|a_{k}\right|\right)<+\infty$, and

$$
L_{a}(z)=(a-z) /(1-\bar{a} z), \quad a \in \Delta, z \in \hat{\mathbf{C}} .
$$

A singular inner function is a function which can be written

$$
S(z)=\exp \left[-\int_{0}^{2 \pi} \frac{e^{i t}+z}{e^{i t}-z} d \mu(t)\right], \quad z \in \Delta,
$$

where $\mu$ is a monotone nondecreasing function on $R$ satisfying $\mu^{\prime}=0$ a.e., $\mu(t+2 \pi)$ $=\mu(t)+\mu(2 \pi)$ and $\mu\left(t^{+}\right)+\mu\left(t^{-}\right)=2 \mu(t), t \in R$. An outer function (of class $\mathfrak{T}$ ) is a function of the form

$$
F(z)=\eta \exp \left(\frac{1}{2 \pi} \int_{0}^{2 \pi} \frac{e^{i t}+z}{e^{i t}-z} \log \psi(t) d t\right], \quad z \in \Delta,
$$

where $|\eta|=1, \psi(t) \geqslant 0$ and $\log \psi(t)$ is integrable.

THEOREM 2.4. Every function $f \in \mathcal{N}$ can be expressed uniquely in the form

$$
f=B\left(S_{1} / S_{2}\right) F,
$$

where $B$ is a Blaschke product, $S_{1}$ and $S_{2}$ are singular inner functions, and $F$ is an outer function (with $\psi(t)=\left|f^{*}\left(e^{i t}\right)\right|$ ). Conversely, every nonconstant function of the form (2.7) belongs to $\mathfrak{R}$. 
In the next definition, we define the Smirnov class $\mathfrak{x}^{+}$, a subclass of $\mathscr{T}$ containing all of the $H^{p}$ spaces, $0<p \leqslant \infty$.

Definition 2.3. A function $f$ is in the class $\mathfrak{R}^{+}$if and only if $f \in \Re$ and $S_{2} \equiv 1$ in the canonical factorization (2.7).

The following characterization of $\mathfrak{x}^{+}$will be of use.

TheOREM 2.5. A function $f \in \mathfrak{X}$ belongs to $\mathfrak{X}^{+}$if and only if

$$
\lim _{s \rightarrow 1} \int_{0}^{2 \pi} \log ^{+}\left|f\left(s e^{i \theta}\right)\right| d \theta=\int_{0}^{2 \pi} \log ^{+}\left|f^{*}\left(e^{i \theta}\right)\right| d \theta
$$

The final class we define in this section is the class of nonconstant inner functions 9 .

DEFINITION 2.4. The class 9 is the class of analytic functions $f$ mapping $\Delta$ into itself, such that $\left|f^{*}(\eta)\right|=1$ for almost all $\eta$ in $C$.

Let $\Im$ (resp. $\delta$ ) denote the class of unimodular multiples of nonconstant Blaschke products (resp. singular inner functions).

THEOREM 2.6. The classes $\mathscr{B}$ and $\delta$ are subclasses of 9 .

3. Components of $\{|f|>r\}$. We start by proving a Phragmén-Lindelöf theorem for continuous subharmonic functions.

THEOREM 3.1. Let $\Phi$ be a region in $\Delta$ and let $u$ be a continuous subharmonic function on $\Phi$. Let $M$ be a real number. If $\lim \sup _{z \rightarrow \zeta} u(z) \leqslant M$ for all $\zeta \in$ fr $\Phi$, except possibly a subset of ( $\mathrm{fr} \Phi) \cap C$ of (linear) measure 0 , and there exists a quasibounded positive harmonic function $h$ on $\Delta$ such that $u(z) \leqslant h(z), z \in \Phi$, then $u(z) \leqslant M, z \in \Phi$.

Recall that a quasibounded positive harmonic function $h$ is a harmonic function which is the limit of a monotone nondecreasing sequence of bounded positive harmonic functions. Also, if $h$ is defined on $\Delta$, and $g$ is an analytic completion of $h$, then $F=\exp \circ g$ is an outer function. On the other hand, if $F$ is an outer function, then $\log |F|=h_{1}-h_{2}$ where $h_{1}$ and $h_{2}$ are quasibounded positive harmonic functions.

Proof. Suppose that there exists $w \in \Phi$ such that $u(w)>M$. Choose $M_{1}$ so that $M<M_{1}<u(w)$ and let $\Omega$ be the component of $\left\{u(z)>M_{1}\right\}$ containing $w$. By the assumed boundary behavior of $u$ at each point of (fr $\Phi) \cap \Delta$, we have (fr $\Omega$ ) $\cap \Delta \subset \Phi$ and $u(\zeta)=M_{1}, \zeta \in($ fr $\Omega) \cap \Delta$. Define

$$
U(z)= \begin{cases}u(z)-M_{1}, & z \in \Omega, \\ 0, & z \in \Delta \backslash \Omega .\end{cases}
$$

Then $U$ is a nonnegative subharmonic function with $U(w)>0$, and $H=h+\left|M_{1}\right|$ is a quasibounded positive harmonic majorant of $U$. 
Let $s \in((1+|w|) / 2,1)$. Since $U$ is subharmonic, we have, using the Poisson integral formula, that

$$
\begin{aligned}
U(w) & \leqslant \frac{1}{2 \pi} \int_{0}^{2 \pi} U\left(s e^{i \theta}\right) \frac{1-|w / s|^{2}}{\left|e^{i \theta}-(w / s)\right|^{2}} d \theta \\
& \leqslant \frac{1}{2 \pi} \int_{0}^{2 \pi} U\left(s e^{i \theta}\right) \frac{s+|w|}{s-|w|} d \theta \\
& <\frac{1}{\pi}\left(\frac{1+|w|}{1-|w|}\right) \int_{0}^{2 \pi} U\left(s e^{i \theta}\right) d \theta .
\end{aligned}
$$

From the definition of $U$ and the assumption that $\limsup _{z \rightarrow \zeta} u(z) \leqslant M$ for almost all $\zeta$ in ( $\mathrm{fr} \Phi) \cap C$, it follows that the radial limit function $U^{*}$ is 0 almost everywhere in $C$. Since $H$ is a quasibounded positive harmonic function, $H=\log |F|$ for some outer function $F$. Therefore, by Theorem 2.5 and the harmonicity of $H$, we have

$$
\int_{0}^{2 \pi} H\left(s e^{i \theta}\right) d \theta=\int_{0}^{2 \pi} H^{*}\left(e^{i \theta}\right) d \theta .
$$

Let $s \rightarrow 1$ in (3.2). Since $0 \leqslant U \leqslant H$ and $U^{*}=0$ a.e., it follows from (3.3) and the Lebesgue dominated convergence theorem that $U(w)=0$, a contradiction. The proof of Theorem 3.1 is thereby completed.

As a consequence of Theorem 3.1 we have the following.

THEOREM 3.2. Let $f \in \mathfrak{R}^{+}$and $r \in(\inf |f|$, sup $|f|)$. If $\Omega$ is a component of $\{|f|>r\}$, then $m[(\mathrm{fr} \Omega) \cap C]>0$.

The notation $m(E)$ is used to denote the linear measure of a measurable subset $E$ of $C$.

Proof. Assume without loss of generality that $r=1$. Let $F$ be the outer factor in the canonical factorization (2.7) of $f$. (Note that $S_{2} \equiv 1$ in that factorization since $f \in \mathfrak{R}^{+}$.) Then the least harmonic majorant $h$ of $\log ^{+}|F|$ is a quasibounded positive harmonic function on $\Delta$ which majorizes the continuous subharmonic function $u=\left.(\log |f|)\right|_{\Omega}$ on $\Omega$. Since $\Omega$ is a component of $\{|f|>1\}$, we must have $|f(\zeta)|=1$, $\zeta \in($ fr $\Omega) \cap \Delta$. If $m[(\operatorname{fr} \Omega) \cap C]=0$, then Theorem 3.1 implies that $|f(z)| \leqslant 1$, $z \in \Omega$, a contradiction. This completes the proof.

Theorem 3.2 does not remain valid for the class $\mathcal{N}$. In fact, for

$$
f(z)=\exp [(1+z) /(1-z)], \quad z \in \Delta,
$$

we have $\{|f|>e\}=\left\{\left|w-\frac{1}{2}\right|<\frac{1}{2}\right\}$ so that $m[(\operatorname{fr}\{|f|>e\}) \cap C]=0$.

We conclude this section with a result for the class $q$ of nonconstant inner functions.

THEOREM 3.3. Let $f \in \mathcal{G}$ and $r \in(0,1)$. If $\Omega$ is a component of $\{|f|>r\}$, then the radius $\overrightarrow{0 \eta}$ is eventually contained in $\Omega$ for almost all $\eta$ in $(\mathrm{fr} \Omega) \cap C$.

For $\eta$ in $C$, we say that the radius $\overrightarrow{0 \eta}$ is eventually contained in $\Omega$ if there exists $t_{\eta} \in(0,1)$ such that $s \eta \in \Omega$ for all $s \in\left[t_{\eta}, 1\right)$. In the proof, we shall use Corollary 3.1 below of the following result of T. Hall $[5$, p. 6]. 
LEMMA 3.1 (HALL). Let $g$ be an analytic mapping of $\{\operatorname{Re} z>0\}$ into $\bar{\Delta}$. Suppose that $r \in(0,1)$ and $\inf \left\{\left|g\left(s e^{i \theta}\right)\right|:-\pi / 2<\theta<\pi / 2\right\} \leqslant r$ for all $s>0$. Then

$$
|g(z)| \leqslant r^{(1 / 3)(1-2 \theta / \pi)},
$$

when $|\operatorname{Arg} z| \leqslant \theta$ and $0 \leqslant \theta<\pi / 2$.

COROLlary 3.1. Let $f$ be an analytic mapping of $\Delta$ into itself and $r \in(0,1)$. If, for some $\varepsilon>0$, we have $\inf \{|f(z)|: z \in \Delta,|z-1|=s\} \leqslant r$ for all $s \in(0, \varepsilon)$, then

$$
\limsup _{t \rightarrow 1}|f(t)| \leqslant r^{1 / 3} \text {. }
$$

Proof of Corollary 3.1. Let $h(z)=\exp [\alpha(z-1) /(z+1)], z \in \Delta$, with $\alpha>0$ chosen so large that $\max \{|h(t)|:-1<t \leqslant 1-\varepsilon\} \leqslant r$. Let $M(z)=(1-z) /(1+z)$, $z \in \hat{\mathbf{C}}$. Then $g(z)=(h f) \circ M(z), \operatorname{Re} z>0$, satisfies the hypotheses of Lemma 3.1, so that $|g(t)| \leqslant r^{1 / 3}, t>0$. Precomposing $g$ with $M$, we have $|(h f)(t)| \leqslant r^{1 / 3}$, $t \in(-1,1)$. Since $\lim _{t \rightarrow 1} h(t)=1$, the inequality (3.5) follows.

We turn now to the proof of Theorem 3.3.

Proof. Since $\left|f^{*}\right|=1$ a.e., there exists a subset $E$ of $(\mathrm{fr} \Omega) \cap C$ satisfying $m(E)=m[(\mathrm{fr} \Omega) \cap C]$ such that the radius $\overrightarrow{0 \eta}$ is eventually contained in some component $\Omega_{\eta}$ of $\{|f|>r\}$ for each $\eta \in E$.

The proof will be complete once it is shown that $E_{\Omega}=\left\{\eta \in E: \Omega_{\eta}=\Omega\right\}$ has the property that $m\left(E \backslash E_{\Omega}\right)=0$. It suffices to show that if $\eta \in E \backslash E_{\Omega}$, then $\left|f^{*}(\eta)\right|=1$ does not hold, for the desired conclusion then follows from the fact that $\left|f^{*}\right|=1$ almost everywhere. To this end, we note that there exists $\varepsilon>0$ such that $\inf \{|f(z)|$ : $|z-\eta|=s\} \leqslant r$ for all $s \in(0, \varepsilon)$. If not, there is a decreasing sequence of positive numbers $\left(s_{n}\right)_{1}^{\infty}$ such that $s_{1}<\varepsilon, \lim _{n \rightarrow \infty} s_{n}=0$, and for every $n$, we have $|f(z)|>r$ when $z \in \Delta$ and $|z-\eta|=s_{n}$. Since $\Omega$ and $\Omega_{\eta}$ are distinct components of $\{|f|>r\}$, at most one of them has nonempty intersection with $\left\{w \in \Delta:|w-\eta|=s_{n}\right\}$ for a given $n$. However, $\eta \in($ fr $\Omega) \cap C$ so that $\Omega \cap\left\{w \in \Delta:|w-\eta|=s_{n}\right\} \neq \varnothing$ for $n$ sufficiently large. Since the same assertion is true for $\Omega_{\eta}$, we have a contradiction. Thus $f(z / \eta)$ fulfills the hypotheses of Corollary 3.1, and it follows that $f$ does not have a radial limit of modulus 1 at $\eta$. The theorem is established.

4. Components of $\{|f|<r\}$. We start this section by showing that Lohwater's property (i), for nonvanishing inner functions (cf. $§ 1$ ), holds for the class $\mathfrak{T}^{+}$.

THEOREM 4.1. Let $f \in \Re^{+}$and $r \in(\inf |f|, \sup |f|)$. If $\Omega$ is a component of $\{|f|<r\}$, then $\Omega$ is a simply-connected Jordan region.

Before stating the proof, we recall some definitions and facts that will be needed.

Definition 4.1. Let $\Phi$ be a region in $\hat{\mathbf{C}}$. Then $\Phi$ is locally connected at $\zeta \in \operatorname{fr} \Phi$ if for each $\varepsilon>0$, there exists $\delta>0$ such that $\{|z-\zeta|<\delta\} \cap \Phi$ is contained in a component of $\{|z-\zeta|<\varepsilon\} \cap \Phi$.

DEFINITION 4.2. If $h$ is a function mapping a region $\Phi$ into $\hat{\mathbf{C}}$, then the cluster set of $h$ at $z \in \bar{\Phi}$, denoted $C(h, z)$, is the set $\bigcap_{t>0} \bar{\Phi}_{t}$, where

$$
\Phi_{t}=h[(\Phi \backslash\{z\}) \cap\{|w-z|<t\}], \quad 0<t<+\infty .
$$

The following result is standard (cf. [2, p. 3]). 
Proposition 4.1. Let $h$ be a continuous function mapping a region $\Phi$ into $\hat{\mathbf{C}}$. If $\Phi$ is locally connected at $\zeta \in \operatorname{fr} \Phi$, then $C(h, \zeta)$ is a continuum or a point.

By definition, a continuum is a compact connected Hausdorff space containing at least two points.

Finally, we quote a result which can be found in [7, p. 135].

Proposition 4.2. Let $\varphi$ be a univalent meromorphic function with domain $\Delta$ and image $\Phi$. If $\Phi$ is locally connected at $\zeta \in \mathrm{fr} \Phi$, then $\lim _{n \rightarrow \zeta} \varphi^{-1}$ exists and is contained in $C$.

We turn now to the proof of Theorem 4.1.

Proof. If ( $\mathrm{fr} \Omega) \cap \Delta$ has a compact component, then the required conclusion follows from Theorem 2.1(iii) and (iv).

Suppose now that (fr $\Omega$ ) $\cap \Delta$ has only noncompact components. By Theorem 2.1(i) we have $\Omega$ is simply-connected, so there exists a Riemann map $\varphi$ of $\Delta$ onto $\Omega$. We shall show that $\Omega$ is a Jordan region by showing that $\varphi$ extends to a homeomorphism of $\bar{\Delta}$ onto $\bar{\Omega}$.

Let $E$ be the subset of fr $\Omega$ consisting of those points which do not have a fr $\Omega$-neighborhood that is a regular analytic Jordan arc. We claim that $E$ is totally disconnected; that is, the components of $E$ are singletons. By Theorem 2.1(ii), the set $E \cap \Delta$ clusters only in $C$, so it suffices to verify the claim for the set $E \cap C$.

We show first that if $\zeta \in E \cap C$, then $\zeta$ is a cluster point of $(\mathrm{fr} \Omega) \cap \Delta$. In fact, since $\zeta \in($ fr $\Omega) \cap C$, there exists $\varepsilon>0$ such that $\Omega \cap\{w \in \Delta:|w-\eta|=s\} \neq \varnothing$ for all $s \in(0, \varepsilon)$. If ( fr $\Omega) \cap \Delta$ does not cluster at $\zeta$, then for some $\delta \in(0, \varepsilon)$ we must have $|f(z)|<r$ for $z \in\{w \in \Delta:|w-\eta|=s\}$ for every $s \in(0, \delta)$. It follows that $\Omega$ contains a $\Delta$-neighborhood of $\eta$, whence there is an open $\operatorname{arc} A$ of $C$ containing $\eta$ which is a fr $\Omega$-neighborhood of $\eta$. This contradicts the assumption that $\zeta \in E$.

To complete the verification of the claim concerning $E$, we show that ( $\mathrm{fr} \Omega) \cap \Delta$ clusters in a subset of $C$ that is nowhere dense. Let $A$ be a nonempty open arc of $C$ such that ( $\operatorname{fr} \Omega) \cap \Delta$ clusters at some point of $A$. By Theorems 2.2 and 2.3 , we have $f \in \mathcal{L}$. Since (fr $\Omega$ ) $\cap \Delta$ has only noncompact components, it follows from Corollary 2.1 and Proposition 2.3 that there exists a noncompact component $l$ of $(\mathrm{fr} \Omega) \cap \Delta$ which clusters in $A$. By Corollary 2.1 and Proposition 2.2, we conclude that $l$ clusters in at most two points. If $l$ clusters only at one point, then $\bar{l}$ is the image of a closed Jordan curve. In this case Theorem 2.1(ii) and Theorem 3.2 imply that $\Omega$ is the bounded component of $\hat{\mathbf{C}} \backslash \bar{l}$ and hence (fr $\Omega$ ) $\cap \Delta$ is not dense in $A$. If $l$ clusters in two points, then $\Delta \backslash l$ is the union of two disjoint Jordan regions $R_{1}$ and $R_{2}$ such that (fr $\left.R_{1}\right) \cap\left(\mathrm{fr} R_{2}\right)=\bar{l}$, and $\Omega$ is contained in precisely one of those regions, say $R_{1}$. Then there is a proper subarc $A_{2}$ of ( $\mathrm{fr} R_{2}$ ) $\cap A$ in which $\Omega$ does not cluster. Thus in this case, we also conclude that (fr $\Omega$ ) $\cap \Delta$ does not cluster in a dense subset of $A$. Since $A$ was arbitrary, it follows that (fr $\Omega) \cap \Delta$ clusters in a nowhere dense subset of $C$. This completes the proof that $E$ is totally disconnected.

We show next that $\varphi^{-1}$ extends analytically to a function $\psi$ defined on $\bar{\Omega} \backslash E$. By the definition of $E$, we have $\Omega$ is locally connected at each point of (fr $\Omega$ ) \E. From Proposition 4.2, it follows that $\lim _{w \rightarrow \zeta} \varphi^{-1}(w)$ exists and is contained in $C$ for each 
$\zeta \in($ fr $\Omega) \backslash E$. The existence of the required analytic extension follows from the Schwarz reflection principle.

We claim now that $\lim _{z \rightarrow \eta} \varphi(z)$ exists for all $\eta$ in $C$. For suppose $\eta \in C$ and the limit does not exist. Then by Proposition 4.1, the cluster set $C(\varphi, \eta)$ is a continuum. Furthermore, since $\varphi$ is a homeomorphism of $\Delta$ onto $\Omega$, the inclusion $C(\varphi, \eta) \subseteq$ fr $\Omega$ must hold. But $E$ is a totally disconnected set, so it cannot contain a continuum. Thus $C(\varphi, \eta)$ clusters at a point of analyticity of $\psi$. Since $\left.\psi\right|_{\Omega}=\varphi^{-1}$, we have $\psi(\zeta)=\eta, \zeta \in C(\varphi, \eta) \backslash E$. Therefore, the identity theorem implies that $\psi$ is constant. This contradicts the fact $\left(\left.\psi\right|_{\Omega}\right)^{-1}=\varphi$. The claim is thereby verified.

Let $\varphi$ be extended to $\bar{\Delta}$ by $\varphi(\eta)=\lim _{z \rightarrow \eta} \varphi(z), \eta \in C$. It is elementary to show that $\varphi$ is continuous on $\bar{\Delta}$ with $\varphi(C) \subseteq$ fr $\Omega$. That $\varphi$ maps $C$ onto fr $\Omega$ is seen as follows. If $\zeta \in(\operatorname{fr} \Omega) \backslash E$, then since $\left.\psi\right|_{\Omega}=\left(\left.\varphi\right|_{\Delta}\right)^{-1}$, we have $\zeta \in C[\varphi, \psi(\zeta)]$ so that $\zeta=\varphi[\psi(\zeta)]$. Since (fr $\Omega) \backslash E$ is dense in fr $\Omega$, and $\varphi(C)$ is closed (by the continuity of $\varphi$ and the compactness of $C$ ), the desired conclusion follows.

It remains only to verify that $\left.\varphi\right|_{C}$ is univalent. If not, there exist $\eta_{1}, \eta_{2} \in C$, $\eta_{1} \neq \eta_{2}$, such that $\varphi\left(\eta_{1}\right)=\varphi\left(\eta_{2}\right)=\zeta \in($ fr $\Omega) \cap C$. Let $K$ be the chord of $C$ with endpoints $\eta_{1}$ and $\eta_{2}$. Now $\varphi(K)$ is the image of a closed Jordan curve and (fr $\Omega) \cap \varphi(K)=\{\zeta\}$. Let $B$ be the bounded component of $\hat{\mathbf{C}} \backslash \varphi(K)$. Since $\Omega$ is open and $\varphi(K) \cap \Delta \subset \Omega$, it follows that $\Omega \cap B \neq \varnothing$. Thus one of the components of $\Delta \backslash K$, call it $R$, has $\varphi(R) \cap B \neq \varnothing$. From the connectedness of $\varphi(R)$ and the fact that $\varphi(R) \cap \varphi(K)=\varnothing$, we conclude that $\varphi(R) \subseteq B$ and hence $\varphi($ fr $R) \subseteq \bar{B}$. Since (fr $R) \cap C$ contains a nondegenerate $\operatorname{arc} A$ of $C, \varphi(C) \subseteq \operatorname{fr} \Omega$ and $(\operatorname{fr} \Omega) \cap \bar{B}=\{\zeta\}$, it follows that $\varphi$ is constant and equal to $\zeta$ on $A$. The Schwarz reflection principle and the identity theorem now imply that $\varphi$ is constant, a contradiction.

Theorem 4.1 is established.

The function $f(z)=\exp [(1+z) /(1-z)], z \in \Delta$, shows that Theorem 4.1 does not hold for the class $\Re$. In fact, $\{|f|<e\}=\Delta \backslash\left\{\left|w-\frac{1}{2}\right|<\frac{1}{2}\right\}$ which is not a Jordan region.

Observing that the reciprocal of an outer function is again an outer function, we have the following corollary of Theorems 3.2 and 4.1.

CoROLlaRY 4.1. Let $f$ be a nonconstant outer function, $r \in(\inf |f|$, $\sup |f|)$, and $\Omega a$ component of $\Delta \backslash \mathcal{L}(f, r)$. Then $\Omega$ is a simply-connected Jordan region for which $m[($ fr $\Omega) \cap C]>0$.

There is an analogue to Corollary 4.1 for a harmonic function $h$ which is the difference of quasibounded positive harmonic functions. This is a consequence of the fact that $h=\log |F|$ for some outer function $F$.

Our next theorem pertains to inner functions.

THEOREM 4.2. Let $f \in \mathcal{R}$. Then $m[(\mathrm{fr} \Omega) \cap C]=0$ for each component $\Omega$ of $\{|f|<r\}$ for every $r \in(\inf |f|, \sup |f|)$, if and only if $f=c g$, where $g \in \mathcal{G}$ and $c \in \mathbf{C} \backslash\{0\}$. 
Proof. Necessity. Let $r \in(\inf |f|$, sup $|f|)$ and let $\Omega_{r}$ be a component of $\{|f|<r\}$. For each $t \geqslant r$, let $\Omega_{t}$ be the unique component of $\{|f|<t\}$ containing $\Omega_{r}$. Then $\left(\Omega_{t}\right.$ : $r \leqslant t<\sup |f|)$ is an ascending family of sets such that

$$
\left\{\left|f^{*}(\eta)\right|<\sup |f|\right\} \subset \bigcup_{r \leqslant t<\sup |f|}\left[\left(\mathrm{fr} \Omega_{t}\right) \cap C\right] .
$$

(Observe that if $\eta \in C$ such that $\left|f^{*}(\eta)\right|<\sup |f|$, then $|f(t \eta)|<M, t \in[0,1)$, for some $M<\sup |f|$.) From (4.1) it follows that

$$
m\left[\left\{\left|f^{*}(\eta)\right|<\sup |f|\right\}\right] \leqslant \lim _{t \rightarrow \sup |f|} m\left[\left(\text { fr } \Omega_{t}\right) \cap C\right] .
$$

By hypothesis, we have $m\left[\left(\operatorname{fr} \Omega_{t}\right) \cap C\right]=0, t \in[r$, sup $|f|)$, so (4.2) implies that $m\left(\left\{\left|f^{*}(\eta)\right|<\sup |f|\right\}\right)=0$. Since $f \in \mathcal{N}$, the radial limit function $f^{*}$ is defined and finite a.e. in $C$ (cf. Theorem 2.3), and we conclude that $\left|f^{*}\right|=\sup |f|$ a.e. and that $\sup |f|<+\infty$. Letting $c=\sup |f|$ and $g=(1 / c) f$, we have $f=c g$ with $g \in \mathscr{9}$ and $c \in \mathbf{C} \backslash\{0\}$.

Sufficiency. We assume, as we may, that $c=1$ so that $f$ is a nonconstant inner function. Let $r \in(0,1)$ and let $\Omega$ be a component of $\{|f|<r\}$. By Corollary 3.1, we have $\lim \sup _{t \rightarrow 1}|f(t \eta)| \leqslant r^{1 / 3}, \eta \in(\operatorname{fr} \Omega) \cap C$. Since $f$ is inner, we have $\left|f^{*}\right|=1$ a.e., and $m[(\mathrm{fr} \Omega) \cap C]=0$ follows. This completes the proof of Theorem 4.2.

Note that in the proof of necessity in Theorem 4.2, we need only require $m[(\operatorname{fr} \Omega) \cap C]=0$ for each component $\Omega$ of $\{|f|<r\}$ for a sequence of $r$ in (inf $|f|$, sup $|f|$ ) converging to sup $|f|$.

Let $f$ be a nonconstant analytic function not of the form $c g$ for $g \in q$ and $c \in \mathbf{C} \backslash\{0\}$. If $m[(\mathrm{fr} \Omega) \cap C]=0$ for every component $\Omega$ of $\{|f|<r\}$ for each $r \in(\inf |f|, \sup |f|)$ (or a sequence of such $r$ converging to sup $|f|$ ), then

$$
\limsup _{t \rightarrow 1}|f(t \eta)|=+\infty
$$

for almost all $\eta$ in $C$ (and inf $|f|=0$ ). However, the converse does not hold. In fact, there exists an analytic function $f$ such that $\lim _{|z| \rightarrow 1}|f(z)|=+\infty, z \in S_{1}$, and $\lim _{|z| \rightarrow 1}|f(z)|=0, z \in S_{2}$, where $S_{k}$ is the spiral $\left\{(-1)^{k} t e^{i[1 /(1-t)]}: 0 \leqslant t<1\right\}$ for $k=1,2$ (cf. [10, p. 336] and [1]). Thus $\lim \sup _{t \rightarrow 1}|f(t \eta)|=+\infty$ for all $\eta$ in $C$, but for a given $r \in(0,+\infty)$ there exists a component $\Omega$ of $\{|f|<r\}$ such that (fr $\Omega) \cap C$ $=C$.

The final result of this section shows that Lohwater's property (ii) (stated in §1) is sharp.

THEOREM 4.3. If $F$ is a nonempty closed subset of $C$ of measure 0 and $r \in(0,1)$, then there exists a singular inner function $S$ and a component $\Omega$ of $\{|S|<r\}$ such that $($ fr $\Omega) \cap C=F$.

For the proof, we shall need a lemma. Suppose that $F$ is an infinite closed set of measure 0 and $\psi: n \rightarrow A_{n}$ is a univalent enumeration of the component arcs of $C \backslash F$ with domain the nonnegative integers. For each $n$, let $\eta_{n}$ denote the "left-hand" endpoint of $A_{n}$, that is, the endpoint with the larger argument in a continuous determination of the argument in $\bar{A}_{n}$. Let $\xi_{n+1} \in\left\{\eta_{k}\right\}_{0}^{n}$ be chosen such that $\mid \eta_{n+1}-$ $\xi_{n+1} \mid=\min _{0 \leqslant k \leqslant n}\left\{\left|\eta_{n+1}-\eta_{k}\right|\right\}$, for each $n$. Finally, let $\sigma_{F}^{\psi}=\sum_{1}^{\infty}\left|\eta_{n}-\xi_{n}\right|$. 
LEMMA 4.1. Given $F$ an infinite closed set of measure 0 , there exists $\psi$ such that $\sigma_{F}^{\psi}<+\infty$.

Proof. We suppose, without loss of generality, that $-1 \notin F$. We shall define a sequence $\left(n_{k}\right)_{1}^{\infty}, 0 \leqslant n_{k}<n_{k+1}$, and corresponding initial sections $\left(A_{j}\right)_{0}^{n_{k}}$ of $\psi$ for each $k$ by induction.

$k=1$. Let $n_{1}=0$ and let $A_{0}$ be the component of $C \backslash F$ containing -1 .

Inductive hypothesis. Let $k \geqslant 1$. Assume that a nonnegative integer $n_{j}$ has been chosen for $1 \leqslant j \leqslant k$, and a component $A_{j}$ of $C \backslash F$ has been chosen for $0 \leqslant j \leqslant n_{k}$ such that $n_{1}=0,-1 \in A_{0}$, and when $k>1$, we have $\left(n_{j}\right)_{1}^{k}$ is strictly increasing, $\left(A_{j}\right)_{0}^{n_{k}}$ is a univalent sequence,

$$
\sum_{0}^{n_{k}} m\left(A_{j}\right) \geqslant 2 \pi\left(1-\frac{1}{2^{k-1}}\right),
$$

and

$$
\sum_{1}^{n_{k}}\left|\eta_{j}-\xi_{j}\right| \leqslant 2 \pi \sum_{0}^{k-2} \frac{1}{2^{j}} .
$$

$k+1$. Since $C \backslash F$ has infinitely many components and $m(C \backslash F)=2 \pi$, there exists $n_{k+1}\left(>n_{k}\right)$ and distinct components $A_{n_{k}+1}, \ldots, A_{n_{k+1}}$ of $C \backslash\left[F \cup\left(\cup_{0}^{n_{k}} A_{j}\right)\right]$ such that (4.3) holds with ' $k+1$ ' replacing ' $k$ '. We assume, as we may, that $\operatorname{Arg} \zeta_{j}<\operatorname{Arg} \zeta_{j+1}$ when $\zeta_{j} \in A_{j}$ and $\zeta_{j+1} \in A_{j+1}$ with $n_{k}+1 \leqslant j<n_{k+1}$.

Now $\cup_{n_{k}+1}^{n_{k+1}} A_{j} \subset C \backslash \cup_{0}^{n_{k}} A_{j}$ and the set $C \backslash \cup_{0}^{n_{k}} A_{j}$ is a finite union of closed arcs of total length $\leqslant 2 \pi / 2^{k-1}$. Furthermore, the "right-hand" endpoint of a nondegenerate component closed arc of $C \backslash \cup_{0}^{n_{k}} A_{j}$ (that is, the endpoint $\zeta$ for which $\operatorname{Arg} \zeta$ is smaller), is equal to $\eta_{j}$ for some $j, 0 \leqslant j \leqslant n_{k}$. Therefore, $\sum_{n_{k}+1}^{n_{k+1}}\left|\eta_{j}-\xi_{j}\right| \leqslant 2 \pi / 2^{k-1}$ and hence (4.4) follows with ' $k+1$ ' replacing ' $k$ '. On noting that the inductive hypothesis is also satisfied for $k=1$, the induction is completed.

By the induction, there exists a univalent enumeration $\psi: n \mapsto A_{n}$ of the component arcs of $C \backslash F$ and a strictly increasing sequence $\left(n_{k}\right)_{1}^{\infty}$ of nonnegative integers such that (4.3) and (4.4) hold for all positive integers $k$. By (4.4), we have $\sigma_{F}^{\psi} \leqslant 4 \pi<+\infty$ as required. This completes the proof of the lemma.

We are now ready to prove the theorem.

Proof. Suppose that $F$ is finite but nonempty, say $F=\left\{\eta_{1}, \ldots, \eta_{n}\right\}$. Let

$$
S_{k}(z)=\exp \left[-\alpha\left(\eta_{k}+z\right) /\left(\eta_{k}-z\right)\right], \quad z \in \Delta,
$$

for $k=1, \ldots, n$, and let $S=\prod_{1}^{n} S_{k}$, where $\alpha=-\log r$. On noting that $t \mapsto\left|S_{k}\left(t \eta_{k}\right)\right|$, $t \in(0,1)$, is a decreasing function of $t$ with $\left|S_{k}(0)\right|=r$ for each $k$, it follows that there is a unique component $\Omega$ of $\{|S|<r\}$ containing all the radii $\overrightarrow{0 \eta_{k}}$. (If $n=1$, the origin is not included.) Since $S$ is a singular inner function which is analytic at each point of $C \backslash F$, we have ( $\mathrm{fr} \Omega$ ) $\cap C=F$, as required.

Suppose now that $F$ is infinite. By Lemma 4.1 there exists $\psi$, a univalent enumeration of the component arcs of $C \backslash F$, such that $\sigma_{F}^{\psi}<+\infty$ (using the notation of the lemma). For each positive integer $k$, let

$$
S_{k}(z)=\exp \left(-\alpha\left|\eta_{k}-\xi_{k}\right|\left(\eta_{k}+z\right) /\left(\eta_{k}-z\right)\right), \quad z \in \Delta,
$$


and

$$
T_{k}(z)=\exp \left(-\alpha\left|\eta_{k}-\xi_{k}\right|\left(\xi_{k}+z\right) /\left(\xi_{k}-z\right)\right), \quad z \in \Delta,
$$

where $\alpha=-\log r$. Let

$$
S=\prod_{1}^{\infty} S_{k} T_{k} .
$$

Since $\sigma_{F}^{\psi}<+\infty$, the function $S$ is a well-defined singular inner function. We shall now prove the following assertion. For each integer $k \geqslant 1$ there exists a nonnegative integer $m_{k}<k$ and a component $\Phi_{k}$ of $\{|S|<r\}$ such that the radii $\overrightarrow{0 \eta_{k}}$ and $\overrightarrow{0 \eta_{m_{k}}}$ are eventually contained in $\Phi_{k}$. Once this assertion is proved, it follows that all of the $\Phi_{k}$ are in fact the same component $\Omega$ of $\{|S|<r\}$ and that $\Omega$ has the property that $\overline{0 \eta_{j}}$ is eventually contained in $\Omega$ for all nonnegative integers $j$. Since $\left\{\eta_{j}\right\}_{0}^{\infty}$ is dense in $F$, and $S$ is analytic at each point of $C \backslash F$, we conclude that (fr $\Omega$ ) $\cap C=F$ as required.

We turn now to the proof of the assertion. Fix $k \geqslant 1$. Let $m_{k}$ be the nonnegative integer such that $\eta_{m_{k}}=\xi_{k}$. By the definition of $S$, it suffices to show that the assertion holds for the product function $S_{k} T_{k}$. Now $\left\{\left|S_{k}\right|<r\right\}$ and $\left\{\left|T_{k}\right|<r\right\}$ are open disks contained in $\Delta$ tangent to $C$ at $\eta_{k}$ and $\xi_{k}=\eta_{m_{k}}$, respectively, such that each has radius $r_{k}=\left|\eta_{k}-\xi_{k}\right| /\left(1+\left|\eta_{k}-\xi_{k}\right|\right)$. The disks have centers $\left(1-r_{k}\right) \eta_{k}$ and $\left(1-r_{k}\right) \xi_{k}$, respectively, so that the distance between their centers is $r_{k}$. On noting that $\left|\left(S_{k} T_{k}\right)(z)\right|<\left|S_{k}(z)\right|,\left|T_{k}(z)\right|, z \in \Delta$, we see that the disks coalesce in taking the product, and the desired conclusion follows. This completes the proof.

In relation to Theorem 4.3 and its proof, the following question arises. Given $r \in(0,1)$ and a nonempty closed set of measure 0 , does there exist an inner function $f$ such that $|f|<r$ on all the radii ending in $F$ ? An affirmative answer would imply the following result. There exists an inner function $g$ and a decreasing function $\mu$ on $[0,1)$ with $\lim _{t \rightarrow 1} \mu(t)=0$, such that $|g(t \eta)| \leqslant \mu(t), t \in[0,1)$, for each $\eta \in F$. Stated another way, uniform convergence to 0 along the radii ending in $F$ is possible for an inner function.

5. The compactness of level sets and their components. In this section we prove the following.

THEOREM 5.1. Let $f$ be a nonconstant analytic function on $\Delta$. Then $\mathcal{L}(f, r)$ is compact for each $r \in(\inf |f|, \sup |f|)$ if and only if $f=c B$, where $B$ is a nonconstant finite Blaschke product and $c \in \mathbf{C} \backslash\{0\}$. If $f \in \mathcal{N}$, then all of the components of $\mathcal{L}(f, r)$ are compact for each $r \in(\inf |f|, \sup |f|)$ if and only if $f=c B$, where $B$ is a nonconstant Blaschke product satisfying

$$
\limsup _{t \rightarrow 1}|B(t \eta)|=1, \quad \eta \in C,
$$

and $c \in \mathbf{C} \backslash\{0\}$.

Proof. Suppose that $r \in(\inf |f|, \sup |f|)$, and $\mathcal{E}(f, r)$ is compact. Let $\Omega$ be a component of $\Delta \backslash \mathcal{L}(f, r)$ which intersects the annulus $\{s<|z|<1\}$, where $s=$ $\sup \{|z|: z \in \mathcal{L}(f, r)\}$. Since ( $\mathrm{fr} \Omega) \cap \Delta \subseteq \mathcal{E}(f, r)$, we conclude that $\Omega$ contains the 
entire annulus. Also, $\Omega$ must be a component of $\{|f|>r\}$. Otherwise, the maximum principle would imply that $|f(z)|<r, z \in \Delta$, which contradicts the assumption on $r$. Since $\Omega$ is a $\Delta$-neighborhood of each point of $C$, we have

$$
\liminf _{z \rightarrow \eta}|f(z)| \geqslant r, \quad \eta \in C .
$$

If $\mathcal{E}(f, r)$ is compact for all $r \in(\inf |f|$, sup $|f|)$, it follows from (5.2) that

$$
\lim _{z \rightarrow \eta}|f(z)|=\sup |f|, \quad \eta \in C .
$$

We claim that $\sup |f|<+\infty$. Otherwise, the meromorphic function $1 / f$ is continuously 0 at each point of $C$, which implies, by the Schwarz reflection principle and the identity theorem, that $1 / f \equiv 0$. This is a contradiction so the claim is verified.

From (5.3) and the fact that sup $|f|<+\infty$, we have $(1 / \sup |f|) f=g$, where $g \in \mathcal{q}$. A theorem of Frostman [4, pp. 107-109] asserts that an inner function which has no radial limit value of 0 is a unimodular multiple of a Blaschke product. Thus (5.3) insures that $g=\eta B$, where $B$ is a (nonconstant) Blaschke product and $|\eta|=1$. It also shows that the zeros of $B$ do not cluster in $C$, so that $B$ is a finite Blaschke product. Hence $f=c B$ with $c=\eta \sup |f| \in \mathbf{C} \backslash\{0\}$ as required.

Sufficiency in the first assertion follows from the fact that a nonconstant finite Blaschke product maps $\Delta$ into itself and its modulus is continuously 1 at each point of $C$.

Suppose now that $f \in \mathcal{R}, r \in(\inf |f|$, sup $|f|)$, and the components of $\mathcal{L}(f, r)$ are compact. Then the components of $\{|f|<r\}$ are relatively compact subsets of $\Delta$. In fact, let $\Omega$ be a component of $\{|f|<r\}$ and let $l$ be a component of (fr $\Omega) \cap \Delta$. If $\Omega$ is not relatively compact, then Theorem 2.1(iii) and (iv) imply that $l$ is noncompact. Therefore, $l$ clusters in $C$ by Corollary 2.1. Since $l \subseteq \mathcal{L}(f, r)$, it follows that $\mathcal{L}(f, r)$ has a noncompact component, contrary to assumption.

It now follows that

$$
\limsup _{t \rightarrow 1}|f(t \eta)| \geqslant r, \quad \eta \in C .
$$

For if (5.4) does not hold, then there exists $\eta \in C$ and a component $\Omega$ of $\{|f|<r\}$ such that radius $\overrightarrow{0 \eta}$ is eventually contained in $\Omega$. However, this contradicts the relative compactness of $\Omega$.

If the components of $\mathcal{L}(f, r)$ are compact for all $r \in(\inf |f|$, sup $|f|)$, we have

$$
\limsup _{t \rightarrow 1}|f(t \eta)|=\sup |f|, \quad \eta \in C \text {. }
$$

Since $f \in \Re$, the function has finite radial limits almost everywhere in $C$ by Theorem 2.3, and it follows from (5.5) that sup $|f|<+\infty$. As before, we conclude that $(1 / \sup |f|) f=\eta B$, where $B$ is a Blaschke product satisfying (5.1) and $|\eta|=1$. Thus $f=c B$, where $c=\eta \sup |f| \in \mathbf{C} \backslash\{0\}$, completing the proof of necessity in the second assertion.

For sufficiency in the second assertion, assume, without loss of generality, that $c=1$. Suppose $\mathcal{L}(f, r)$ has a noncompact component $l$ for some $r \in(0,1)$. Then $l$ clusters at some point $\eta \in C$. By Corollary 3.1, we conclude that $\lim _{\sup _{t \rightarrow 1}}|f(t \eta)| \leqslant$ $r^{1 / 3}<1$. This completes the proof of sufficiency. 
Theorem 5.1 is established.

Note that in the proof of necessity in the first (resp. second) assertion of Theorem 5.1 , it suffices that there exists a sequence of $r$ in $(\inf |f|, \sup |f|)$ converging to $\sup |f|$ such that $\mathcal{L}(f, r)$ is compact (resp. has all its components compact).

Let $f$ be a nonconstant analytic function not contained in $\mathscr{N}$. If $\varrho(f, r)$ has compact components for every $r \in(\inf |f|$, sup $|f|$ ) (or a sequence of such $r$ converging to $\sup |f|)$, then it follows from arguments given above that $\limsup _{t \rightarrow 1}|f(t \eta)|=$ $+\infty$ for all $\eta$ in $C$. The converse does not hold as the example following the proof of Theorem 4.2 shows.

6. Level set uniqueness theorems. The first result of this section is a uniqueness theorem for functions in the class 4 .

Theorem 6.1. Let $f, g \in \Psi$ and $r, s \in(0,1)$. Then $\mathcal{L}(f, r)=\mathfrak{L}(g, s)$ if and only if $f$ has an analytic $\alpha$ th power $f^{\alpha}$ and $g=\eta f^{\alpha}$, where $\alpha=(\log s) / \log r$ and $|\eta|=1$.

The following pertinent observations are classical. If $f$ is meromorphic on $\Delta$ without poles or zeros, then $f$ has an analytic $\alpha$ th power for all $\alpha \in(-\infty, \infty)$. If $f$ has a zero or a pole but is not identically 0 , then for $\alpha \in(-\infty,+\infty)$, there exists a meromorphic $\alpha$ th power of $f$ if and only if $\alpha$ is of the form $n / d$, where $n$ is an integer and $d$ is the greatest common divisor of the numbers in the set $\left\{\left|\partial_{f}(a)\right|: \partial_{f}(a) \neq 0\right\}$. For $a \in \Delta$, we use $\partial_{f}(a)$ to denote the divisor of $f$ at $a$, that is, $\inf \left\{k: b_{k} \neq 0\right\}$, where $\sum_{-\infty}^{\infty} b_{k}(z-a)^{k}$ is the Laurent expansion of $f$ around $a$.

Before giving the proof of Theorem 6.1, we shall prove a proposition. For the proposition, assume $f \in \mathscr{G}, r \in(0,1)$, and $\Omega$ a component of $\{|f|>r\}$ are all fixed, and let $\mathcal{H}_{\Omega}$ be the collection of all harmonic functions $u$ with domain $\Omega$ which satisfy

$$
0 \leqslant u(z) \leqslant 1, \quad z \in \Omega,
$$

and

$$
\lim _{z \rightarrow \zeta} u(z)=0, \quad \zeta \in(\text { fr } \Omega) \cap \Delta .
$$

Furthermore, for $g \in \Phi$, define

$$
H_{g}(z)=\log |g(z) / r| / \log (1 / r), \quad z \in \Omega .
$$

Proposition 6.1. The maximum function in $\mathcal{H}_{\Omega}$ is $H_{f}$.

In the proof of Proposition 6.1, we shall use Corollary 6.1 below of the following theorem [4, pp. 111-112].

Theorem 6.2 (Frostman). Let $g \in 9$. Then

$$
E=\left\{a \in \Delta: L_{a} \circ g \notin \Re\right\}
$$

has zero (inner) capacity.

Recall that 93 is defined to be the class of unimodular multiples of nonconstant Blaschke products. We remark that in the original theorem of Frostman cited above, it was proved that $E$ is a set of inner capacity 0 . Heins [6, p. 448] showed that $E$ is an $\widetilde{F}_{\sigma}$ set, whence $E$ is actually of capacity 0 . We shall only need the following corollary 
of the original theorem. We state it without proof since it can be proved without difficulty from Theorem 6.2 and the definition of inner capacity.

Corollary 6.1. Let $g \in \mathscr{G}$ and let $E$ be defined by (6.4). Then $\Delta \backslash E$ is a dense subset of $\Delta$.

We turn now to the proof of Proposition 6.1.

Proof. By Corollary 6.1, there exists a sequence $\left(a_{k}\right)_{1}^{\infty}$ with $\lim _{k \rightarrow \infty} a_{k}=0$, such that for each $k$ we have $\left|a_{k}\right|<r / 2$ and $B_{k} \equiv-L_{a_{k}} \circ f \in \Re$. Since $\left(L_{a_{k}}\right)_{1}^{\infty}$ converges uniformly on all of $\Delta$ to the function $-z$, it follows that $\left(B_{k}\right)_{1}^{\infty}$ is a sequence of functions converging uniformly on all of $\Delta$ to $f$. In addition, since $\left|a_{k}\right|<r / 2$ and $\Omega$ is a component of $\{|f|>r\}$, we have $B_{k}$ is bounded away from 0 on $\Omega$ for each $k$.

Define

$$
\varepsilon_{k}=\sup _{z \in \Omega}\left|H_{B_{k}}(z)-H_{f}(z)\right|, \quad k=1,2, \ldots
$$

By the uniform convergence of $\left(B_{k}\right)_{1}^{\infty}$ to $f$ on $\Delta$, the uniform continuity of log on compact subsets of $(0,+\infty)$, and the fact that $f$ and the $B_{k}$ are bounded away from 0 and $\infty$ on $\Omega$, we have $0 \leqslant \varepsilon_{k}<+\infty$ for each $k$ and $\lim _{k \rightarrow \infty} \varepsilon_{k}=0$.

Let $\left(\Omega_{k}\right)_{1}^{\infty}$ be a sequence of subregions of $\Omega$ satisfying $\bar{\Omega}_{k} \subset \Omega_{k+1}$ for each $k$, and $\cup_{1}^{\infty} \Omega_{k}=\Omega$. Owing to the uniform product convergence of Blaschke products on compact subsets of $\Delta$, there exists for each $k$ a finite subproduct $b_{k}$ of $B_{k}$ such that

$$
\left|b_{k}(z)-B_{k}(z)\right| \leqslant 1 / 2^{k}, \quad z \in \bar{\Omega}_{k} .
$$

By the convergence of $\left(B_{k}\right)_{1}^{\infty}$ to $f$ and (6.6), we conclude that $\lim _{k \rightarrow \infty} b_{k}=f$. Since $\left|b_{k}(z)\right| \geqslant\left|B_{k}(z)\right|>0, z \in \Omega$, it follows that

$$
h_{k}=H_{b_{k}}+\varepsilon_{k}
$$

is harmonic on $\Omega$ for each $k$ with

$$
\lim _{k \rightarrow \infty} h_{k}=H_{f} \text {. }
$$

Futhermore, by (6.5), the monotonicity of $\log$, and the fact that $\left|b_{k}(z)\right| \geqslant\left|B_{k}(z)\right|$, we conclude that

$$
h_{k} \geqslant H_{f}, \quad k=1,2, \ldots
$$

We show now that $h_{k} \geqslant u$ for every $u \in \mathcal{H}_{\Omega}$ and each $k$. Let $k$ be a positive integer. Since $b_{k}$ is a unimodular multiple of a finite Blaschke product, it is continuous and of modulus 1 at each point of $C$. In particular, $\lim _{z \rightarrow \eta} h_{k}(z)=1+\varepsilon_{k}$, $\eta \in(\mathrm{fr} \Omega) \cap C$. On the other hand, from (6.9) and the fact that $H_{f}>0$, it follows that $\lim _{z \rightarrow \zeta} h_{k}(z) \geqslant 0, \zeta \in(\mathrm{fr} \Omega) \cap \Delta$. (Note that the limit does exist.) If $u \in \mathcal{H}_{\Omega}$, then by (6.1) and (6.2) along with the boundary maximum principle for harmonic functions, we conclude that $h_{k} \geqslant u$ as we set out to show.

It follows from (6.8) and the result just obtained, that

$$
H_{f} \geqslant u, \quad u \in \mathcal{H}_{\Omega} .
$$


It remains only to verify that $H_{f} \in \mathfrak{H}_{\Omega}$. By the definition of $\Omega$ and the fact that $|f(z)|<1, z \in \Delta$, the function $H_{f}$ satisfies (6.1). Since $|f(\zeta)|=r, \zeta \in($ fr $\Omega) \cap \Delta$, we see that (6.2) is satisfied. Thus $H_{f} \in \mathcal{H}_{\Omega}$, and $H_{f}$ is the maximum function in $\mathcal{H}_{\Omega}$.

We are now ready to prove Theorem 6.1.

Proof. Sufficiency. Suppose that $f$ has an analytic $\alpha$ th power $f^{\alpha}$ and $g=\eta f^{\alpha}$ for some $\eta \in C$. Then $\mathcal{L}^{2}(g, s)=\{|g|=s\}=\left\{|f|^{\alpha}=s\right\}=\{|f|=r\}=\mathcal{E}(f, r)$, using the fact that $s^{1 / \alpha}=r$.

Necessity. Suppose that $\mathcal{L}(f, r)=\mathscr{L}(g, s)$. Let $\Omega$ be a component of $\{|f|>r\}$. By Theorem 3.2, we have $m[(\mathrm{fr} \Omega) \cap C]>0$. Theorem 4.2 implies that if $\Omega$ were a component of $\{|g|<s\}$, then $m[(\operatorname{fr} \Omega) \cap C]=0$, so we conclude that $\Omega$ is a component of $\{|g|>s\}$. Let $H_{f}$ be defined by (6.3) with $f$ replacing $g$, and let $H_{g}$ be given by (6.3) with $s$ replacing $r$. By Proposition 6.1, we have $H_{f}=H_{g}$, or equivalently,

$$
\alpha \log |f(z)|=\log |g(z)|, \quad z \in \Omega .
$$

The proof of necessity is completed on applying the following.

Lemma 6.1. Let $f$ and $g$ be nonconstant meromorphic functions on $\Delta$. Suppose that $\alpha \in(-\infty, \infty)$ with $\alpha \neq 0$ and $\Omega$ is an open subset of $\Delta$ such that (6.11) holds. Then $g=\eta f^{\alpha}$, where $f^{\alpha}$ is a meromorphic $\alpha$ th power of $f$ and $|\eta|=1$.

Proof. Let $E$ be the set of zeros and poles of $f$ and $g$. By (6.11), the identity theorem for harmonic functions, the continuity of $\log |f|$ and $\log |g|$ (in the extended sense), and the isolated character of the set $E$, we conclude that

$$
\alpha \log |f(z)|=\log |g(z)|, \quad z \in \Delta .
$$

We claim now that

$$
\alpha \partial_{f}(z)=\partial_{g}(z), \quad z \in \Delta .
$$

If $a \in \Delta \backslash E$, then by the definitions of $E$ and $\partial$, we have $\partial_{f}(a)=\partial_{g}(a)=0$. On the other hand, if $a \in E$, then (6.12) insures that both $\partial_{f}(a)$ and $\partial_{g}(a)$ are nonzero. In this case,

$$
f(z)=(z-a)^{\partial_{f}(a)} f_{1}(z) \text { and } g(z)=(z-a)^{\partial_{g}(a)} g_{1}(z), \quad z \in D \backslash\{a\},
$$

where $D$ is an open disk with center $a$ on which $f_{1}$ and $g_{1}$ are nonvanishing analytic functions. From (6.12), we conclude that

$$
\left[\alpha \partial_{f}(a)-\partial_{g}(a)\right] \log |z-a|=\log \left|g_{1}(z)\right|-\alpha \log \left|f_{1}(z)\right|, \quad z \in D \backslash\{a\} .
$$

On considering the limiting behavior as $z$ approaches $a$, we have $\alpha \partial_{f}(a)=\partial_{g}(a)$, and the claim is verified.

We show next that $f$ has an analytic $\alpha$ th power $f^{\alpha}$. If $E=\varnothing$, then $f$ is a nonvanishing analytic function. If $E \neq \varnothing$, then it follows from (6.13) that $\alpha=n / d$ where $d$ is the greatest common divisor of the numbers in the set $\left\{\left|\partial_{f}(a)\right|\right.$ : $\left.\partial_{f}(a) \neq 0\right\}$ and $n$ is a nonzero integer. Thus $f$ also has a meromorphic $\alpha$ th power $f^{\alpha}$ in either case (cf. the paragraph following the statement of Theorem 6.1). 
By (6.12), we have

$$
\left|g / f^{\alpha}\right|=\exp (\log |g|-\alpha \log |f|)=1, \quad z \in \Delta \backslash E .
$$

Thus it follows from the maximum principle that $g=\eta f^{\alpha}$, where $|\eta|=1$. This completes the proof of the lemma.

RemarKs. (1) If $f \in \mathscr{G}$ and $g$ is a nonconstant outer function, then $\mathcal{L}(f, r) \neq \mathscr{L}(g, s)$ for any allowed $r$ and $s$. In fact, a component $\Omega$ of $\{|f|<r\}$ satisfies $m[($ fr $\Omega) \cap C]$ $=0$ by Theorem 4.2. However, every component $\Phi$ of $\Delta \backslash \mathcal{L}(g, s)$ has $m[(\mathrm{fr} \Phi) \cap C]$ $>0$ by Corollary 4.1 .

(2) If $f \in \mathcal{G}$ and $g$ is a nonconstant bounded analytic function with $\mathscr{L}(f, r)=$ $\mathcal{L}(g, s)$, we cannot draw the conclusion that $g=c f^{\alpha}$ where $\alpha>0, f$ is an analytic $\alpha$ th power of $f$, and $c \in \mathbf{C} \backslash\{0\}$. To see this, it suffices to consider the simplest example, namely $f(z)=z, z \in \Delta$. In fact, if $B$ is any finite Blaschke product with all of its zeros contained in $\{|z|<r\}$, then $g(z)=s B(z / r)$ is a bounded analytic function with $\varrho(f, r)=\mathscr{L}(g, s)$. This example also shows that the following theorem is sharp.

THEOREM 6.3. Let $f \in 9$ and let $g$ be an arbitrary nonconstant meromorphic function on $\Delta$. Then there exist $r_{1}, r_{2} \in(0,1), r_{1} \neq r_{2}$, and $s_{1}, s_{2} \in(\inf |g|$, sup $|g|)$ such that $\mathfrak{L}\left(f, r_{k}\right)=\mathcal{L}\left(g, s_{k}\right), k=1,2$, if and only if $g=c f^{\alpha}$, where $\alpha \in(-\infty, \infty), \alpha \neq 0, f^{\alpha}$ is a meromorphic $\alpha$ th power of $f$, and $c \in \mathbf{C} \backslash\{0\}$.

Proof. Sufficiency is clear so we prove only necessity.

Without loss of generality, assume that $r_{1}<r_{2}$. Let $\Omega$ be a component of $\left\{|f|<r_{2}\right\}$. By Theorems 4.1 and 4.2 , we have $\Omega$ is a simply-connected Jordan region for which $m[(\mathrm{fr} \Omega) \cap C]=0$. Let $\varphi$ be a Riemann map of $\Delta$ onto $\Omega$. Since $\Omega$ is a Jordan region, $\varphi$ extends to a homeomorphism of $\bar{\Delta}$ onto $\bar{\Omega}$. From a standard theorem concerning the measure of boundary sets under conformal enlargement (cf. [2, p. 54]) along with the fact $m[(\mathrm{fr} \Omega) \cap C]=0$, we have $m\left\{\varphi^{-1}[(\mathrm{fr} \Omega) \cap C]\right\}=0$. Furthermore $|f(\eta)|=r_{2}, \eta \in(\operatorname{fr} \Omega) \cap \Delta$, so that $|f \circ \varphi(\eta)|=r_{2}, \eta \in \varphi^{-1}[(\operatorname{fr} \Omega) \cap \Delta]$. Thus $f_{*}=\left(1 / r_{2}\right) f \circ \varphi$ is a nonconstant inner function. In addition,

$$
\varphi^{-1}\left[\mathcal{L}\left(f, r_{1}\right) \cap \Omega\right]=\varrho\left(f_{*}, r_{1} / r_{2}\right) .
$$

Assume first that $s_{1}<s_{2}$. Since $\Omega$ is a component of $\Delta \backslash \mathcal{L}\left(g, s_{2}\right)$ with $\Omega \cap \mathcal{L}\left(g, s_{1}\right)$ $=\Omega \cap \mathcal{L}\left(f, r_{1}\right) \neq 0$, it follows that $\Omega$ is a component of $\left\{|g|<s_{2}\right\}$. As above, we see that $g_{*}=\left(1 / s_{2}\right) g \circ \varphi$ is a nonconstant inner function with $\varphi^{-1}\left[\mathcal{L}\left(g, s_{1}\right) \cap \Omega\right]=$ $\mathcal{L}\left(g_{*}, s_{1} / s_{2}\right)$. Also, the equality $\mathcal{L}\left(f, r_{1}\right)=\mathcal{L}\left(g, s_{1}\right)$ insures that $\mathcal{L}\left(f_{*}, r_{1} / r_{2}\right)=$ $\mathcal{L}\left(g_{*}, s_{1} / s_{2}\right)$. By Theorem 6.1, we have $g_{*}=\eta f_{*}^{\alpha}$, where

$$
\alpha=\log \left(s_{1} / s_{2}\right) / \log \left(r_{1} / r_{2}\right),
$$

$f_{*}^{\alpha}$ is an analytic $\alpha$ th power of $f_{*}$, and $|\eta|=1$. Thus

$$
\log \left|\left(r_{2}^{\alpha} / s_{2}\right) g(z)\right|=\alpha \log |f(z)|, \quad z \in \Omega,
$$

and it follows from Lemma 6.1 that $g=c f^{\alpha}$, where $f^{\alpha}$ is an analytic $\alpha$ th power of $f$ and $c \in \mathbf{C} \backslash\{0\}$. 
The case $s_{1}>s_{2}$ is reduced to the case just treated by considering $1 / g$. This completes the proof.

The proof of necessity in Theorem 6.3 depends only on the property that $\Delta \backslash \mathcal{L}\left(f, r_{2}\right)$ has a component $\Omega$ which is a simply-connected Jordan region with $m[(\operatorname{fr} \Omega) \cap C]=0$. We, therefore, have the following corollaries.

COROLlaRY 6.2. Let $f$ and $g$ be nonconstant analytic and meromorphic functions on $\Delta$, respectively. Suppose that

$$
r_{1}, r_{2} \in(\inf |f|, \sup |f|), \quad r_{1}<r_{2}, \quad \text { and } s_{1}, s_{2} \in(\inf |g| \text {, sup }|g|) .
$$

If $\mathcal{L}\left(f, r_{k}\right)=\mathfrak{L}\left(g, s_{k}\right), k=1,2$, and $\mathcal{L}\left(f, r_{2}\right)$ has a compact component, then $g=c f^{\alpha}$, where $\alpha \in(-\infty, \infty), \alpha \neq 0, f^{\alpha}$ is a meromorphic $\alpha$ th power of $f$, and $c \in \mathbf{C} \backslash\{0\}$.

COROLlARY 6.3. Let $f$ and $g$ be nonconstant meromorphic functions on $\Delta$ such that $f$ has a zero. Suppose that there exist sequences $\left(r_{n}\right)_{1}^{\infty}$ in $(0, \sup |f|)$ with $\lim _{n \rightarrow \infty} r_{n}=0$, and $\left(s_{n}\right)_{1}^{\infty}$ in (inf $|g|$, sup $\left.|g|\right)$ such that $\mathcal{E}\left(f, r_{n}\right)=\mathcal{L}\left(g, s_{n}\right)$ for each $n$. Then $g=c f^{\alpha}$, where $\alpha \in(-\infty, \infty), \alpha \neq 0, f^{\alpha}$ is a meromorphic $\alpha$ th power of $f$, and $c \in \mathbf{C} \backslash\{0\}$.

Corollary 6.3 is not valid when the assumption that $f$ has a zero is replaced by $\inf |f|=0$, even when both $f$ and $g$ are bounded.

EXAMPLE 6.1. Let $\nu$ be a continuous monotone nondecreasing function on $[0,1]$ with $\nu(0)=0$ and $\nu(1)=1$. Let $u$ be the unique harmonic function on $R=\{0<$ $\operatorname{Re} z<1,0<\operatorname{Im} z<1\}$ with a continuous extension $U$ on $\bar{R}$ satisfying

$$
U(z)= \begin{cases}0, & z=t, \\ 1, & z=t+i, \\ \nu(t), & z=i t, \\ t, & z=1+i t,\end{cases}
$$

for $0 \leqslant t \leqslant 1$. (The extended function $U$ is the unique solution to the Dirichlet problem for $R$ with boundary function (6.16).) By reflection, we can define a harmonic function $v$ on $S=\{0<\operatorname{Re} z<1, \operatorname{Im} z>0\}$ with $\left.v\right|_{R}=u$. The function $v$ extends continuously to a function $V$ on $\bar{S}$ such that $\left.V\right|_{R}=U$ and

$$
V(z)=2 n-V[\operatorname{Re} z+i(2 n-\operatorname{Im} z)],
$$

for $n \leqslant \operatorname{Im} z \leqslant n+1,0 \leqslant \operatorname{Re} z \leqslant 1$, when $n$ is a positive integer. In particular, since $0<V(z)<1, z \in R$, and $V(t)=0, V(t+i)=1,0 \leqslant t \leqslant 1$, it follows that $\{z \in S$ : $V(z)=n\}=\{\operatorname{Im} z=n, 0<\operatorname{Re} z<1\}$ for each positive integer $n$.

Let $\varphi$ be a Riemann map of $\Delta$ onto $S$. Then $\varphi$ extends to a homeomorphism of $\bar{\Delta}$ onto $\bar{S}$ since $S$ is a Jordan region in $\hat{\mathbf{C}}$. Furthermore, the function $H=V \circ \varphi$ is continuous on $\bar{\Delta}$, harmonic in $\Delta$, and satisfies

$$
H\left[\varphi^{-1}(i t)\right]=V(i t)=\nu(t), \quad 0 \leqslant t \leqslant 1 .
$$

Let $g$ be an analytic completion of $h=\left.H\right|_{\Delta}$ and let $f_{\nu}=\exp (-g)$. Then

$$
\mathcal{L}\left(f_{\nu}, e^{-n}\right)=\varphi^{-1}[\{\operatorname{Im} z=n, 0<\operatorname{Re} z<1\}]
$$

for each positive integer $n$, and $\left|f_{\nu}\right|$ is continuously equal to $\exp [-\nu(t)]$ at $\varphi^{-1}($ it $)$ for $0 \leqslant t \leqslant 1$. Thus, for allowed but unequal $\nu_{1}$ and $\nu_{2}$, we have $\mathcal{L}\left(f_{\nu_{1}}, e^{-n}\right)=\mathcal{L}\left(f_{\nu_{2}}, e^{-n}\right)$ for each positive integer $n$, but $f_{\nu_{1}} \neq c\left(f_{\nu_{2}}\right)^{\alpha}$ for $\alpha \in(-\infty, \infty)$ and $c \in \mathbf{C} \backslash\{0\}$. 
Before stating our last theorem in full generality, we prove a restricted form of it as a lemma.

Lemma 6.2. Let $f \in \mathfrak{N}^{+}$. If, for a sequence $\left(r_{n}\right)_{1}^{\infty}$ in $(\inf |f|$, sup $|f|)$ converging to $\sup |f|$, there exist sequences $\left(f_{n}\right)_{1}^{\infty}$ in 9 and $\left(s_{n}\right)_{1}^{\infty}$ in $(0,1)$ such that $\mathcal{L}\left(f, r_{n}\right)=$ $E\left(f_{n}, s_{n}\right)$ for each $n$, then $f=c g$ for some $g \in \mathscr{G}$ and $c \in \mathbf{C} \backslash\{0\}$.

Proof. We start with some general observations. If $\mathcal{L}=\mathcal{L}(G, r)$ for some noncon-

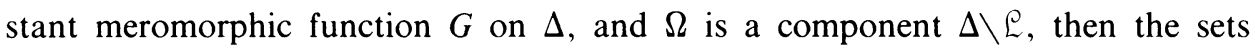
$\{|G|>r\}$ and $\{|G|<r\}$ are uniquely determined by $\mathcal{L}$ and the knowldege of which of these sets $\Omega$ is a subset. This is a consequence of the local behavior of $G$ as given in Proposition 2.1. Thus if $G_{1}$ and $G_{2}$ are nonconstant meromorphic functions on $\Delta$ with $\mathcal{L}\left(G_{1}, r\right)=\mathcal{L}\left(G_{2}, s\right)$, then either $\left\{\left|G_{1}\right|>r\right\}=\left\{\left|G_{2}\right|>s\right\}$ or $\left\{\left|G_{1}\right|<r\right\}=$ $\left\{\left|G_{2}\right|>s\right\}$.

Now suppose $n$ is a positive integer. We claim that $\left\{|f|>r_{n}\right\}=\left\{\left|f_{n}\right|>s_{n}\right\}$. Otherwise, $\left\{|f|<r_{n}\right\}=\left\{\left|f_{n}\right|>s_{n}\right\}$. Since $f_{n} \in 9$, almost all radii $\overrightarrow{0 \eta}$ are eventually contained in $\left\{\left|f_{n}\right|>s_{n}\right\}$, and we conclude that $\limsup _{t \rightarrow 1}|f(t \eta)| \leqslant r_{n}$ for almost all $\eta$ in $C$. However, this implies that $\sup |f| \leqslant r_{n}$ since $f \in \mathfrak{T}^{+}$(cf. Definition 2.3), contradicting the fact that $r_{n} \in(\inf |f|, \sup |f|)$. The claim is verified. It also follows from the argument just given, that $\liminf _{t \rightarrow 1}|f(t \eta)| \geqslant r_{n}$ for almost all $\eta$ in $C$.

From the hypotheses and the preceding paragraph, we now conclude that $\left|f^{*}\right|=$ $\sup |f|$ a.e. and $\sup |f|<+\infty$. Thus

$$
g=(1 / \sup |f|) f \in \mathcal{G} \text { and } f=c g
$$

with $c=\sup |f| \in \mathbf{C} \backslash\{0\}$ as required.

For the following theorem, let $C$ be a subclass of 9 with the property that whenever $f \in \mathcal{C}$ has an analytic positive power $f^{\alpha}$, we have $f^{\alpha} \in \mathcal{C}$. (For example, $\mathcal{C}$ can be taken to be $9, \Re$ or $\mathcal{S}$.)

THEOREM 6.4. Let $f \in \Re^{+}$. If, for a sequence $\left(r_{n}\right)_{1}^{\infty}$ in (inf $|f|$, sup $\left.|f|\right)$ converging to

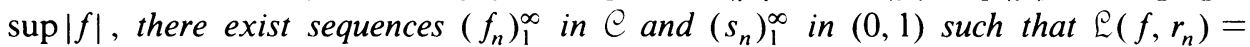
$\mathcal{L}\left(f_{n}, s_{n}\right)$, then $f=c g$ for some $g \in \mathcal{C}$ and $c \in \mathbf{C} \backslash\{0\}$.

Proof. By Lemma 6.2, we have $f=c_{1} g_{1}$ for some $g_{1} \in 9$ and $c_{1} \in \mathbf{C} \backslash\{0\}$. From the assumption on $f$, it follows that $g_{1}$ shares a level set of its modulus with a function in the class $e$. We conclude from Theorem 6.1 that $g_{1}=c_{2}\left(g_{2}\right)^{\alpha}$, where $\alpha>0,\left(g_{2}\right)^{\alpha}$ is an analytic $\alpha$ th power of $g_{2} \in \mathcal{C}$, and $c_{2} \in C$. Since $C$ contains the analytic positive powers of its functions, we have $g=\left(g_{2}\right)^{\alpha} \in \mathcal{C}$. Thus $f=c g$ where $c=c_{1} c_{2} \in \mathbf{C} \backslash\{0\}$ and $g \in \mathcal{C}$, completing the proof.

The example $f(z)=\exp [(1+z) /(1-z)], z \in \Delta$, shows that Theorem 6.4 is not valid for all $f \in \mathfrak{X}$. However, for special classes $\mathcal{C}$, the requirement $f \in \mathfrak{X}^{+}$of Theorem 6.4 can be weakened. It is immediate from Theorem 5.1 and the remark directly following it, that Theorem 6.4 remains valid for $f$ an arbitrary nonconstant analytic function (resp. $f \in \mathcal{N}$ ) when $\mathcal{C}$ is the class of nonconstant finite Blaschke products (resp. the class of nonconstant Blaschke products satisfying (5.1)). 


\section{REFERENCES}

1. F. Bagemihl and W. Seidel, Some houndary properties of analytic functions, Math. Z. 61 (1954), $186-199$

2. E. F. Collingwood and A. J. Lohwater, The theory of cluster sets, Cambridge Univ. Press, London, 1966.

3. P. Duren, Theory of $H^{p}$ spaces, Academic Press, New York, 1970.

4. O. Frostman, Potential d'équilibre et capacité des ensembles avec quelques applications á la théorie des fonctions, Thése, Lund, 1935.

5. T. Hall, Sur la mesure harmonique de certains ensembles, Ark. Mat. Astr. Fys. 25A (1937), 8pp.

6. M. Heins, On the Lindelöf principle, Ann. of Math. (2) 61 (1955), 440-473.

7. Selected topics in the classical theory of functions of a complex variable, Holt, New York, 1962.

8. A. J. Lohwater, The reflection principle and the distribution of values of functions defined in a circle, Amer. Acad. Sci. Fenn. Ser. AI 229 (1956), 18 pp.

9. G. R. MacLane, Asymptotic values of holomorphic functions, Rice Univ. Studies 1 (1963).

10. W. J. Schneider, Approximation and harmonic measure, Aspects of Contemporary Complex Analysis (D. H. Brannan and J. G. Clunie, eds.), Academic Press, New York, 1980, pp. 334-340.

Department of Mathematics, Wayne State University, Detroit, Michigan 48202 